\title{
Long-term outcome of profound hyponatremia: a prospective 12 months follow-up study
}

\author{
Bettina Winzeler ${ }^{1,3, *}$, Nica Jeanloz ${ }^{1, *}$, Nicole Nigro ${ }^{1,3, *}$, Isabelle Suter-Widmer ${ }^{1,3}$, \\ Philipp Schuetz ${ }^{2,3}$, Birsen Arici', Martina Bally ${ }^{2,3}$, Claudine Blum ${ }^{2,3}$, Andreas Bock ${ }^{3,4}$, \\ Andreas Huber ${ }^{3,5}$, Beat Mueller ${ }^{2,3}$ and Mirjam Christ-Crain ${ }^{1,3}$ \\ ${ }^{1}$ Endocrinology, Diabetology and Metabolism, University Hospital Basel, Basel, Switzerland, ${ }^{2}$ Division of \\ Endocrinology, Diabetology and Metabolism, Medical University Clinic, Kantonsspital Aarau, Aarau, Switzerland, \\ ${ }^{3}$ Department of Clinical Research, University Hospital Basel, Basel, Switzerland, ${ }^{4}$ Nephrology, Dialysis \& \\ Transplantation, Kantonsspital Aarau, Aarau, Switzerland, and 5 Institute of Laboratory Medicine, \\ Kantonsspital Aarau, Aarau, Switzerland \\ *(B Winzeler, N Jeanloz and N Nigro contributed equally to this work)
}

\author{
Correspondence \\ should be addressed \\ to B Winzeler \\ Email \\ Bettina.winzeler@usb.ch
}

\begin{abstract}
Background: Hyponatremia is the most common electrolyte abnormality in hospitalized patients and given its impact on mortality and morbidity, a relevant medical condition. Nevertheless, little is known about factors influencing longterm outcome.

Methods: This is a prospective observational 12-month follow-up study of patients with profound hyponatremia ( $\leq 125 \mathrm{mmol} / \mathrm{L}$ ) admitted to the emergency department of two tertiary care centers between 2011 and 2013 . We analyzed the predictive value of clinical and laboratory parameters regarding the following outcomes: 1-year mortality, rehospitalization and recurrent profound hyponatremia.

Results: Median (IQR) initial serum sodium (s-sodium) level of 281 included patients was 120 mmol/L (116-123). During the follow-up period, 58 (20.6\%) patients died. The majority $(56.2 \%)$ were rehospitalized at least once. Recurrent hyponatremia was observed in $42.7 \%$, being profound in $16 \%$. Underlying comorbidities, assessed by the Charlson Comorbidity Index, predicted 1-year mortality (odds ratio (OR) $1.43,95 \%$ confidence interval $(\mathrm{Cl}) 1.25-1.64, P<0.001)$. Furthermore, 's-sodium level at admission' (OR 1.14, 95\% Cl 1.01-1.29, $P=0.036$ ) and 'correction of hyponatremia' defined as s-sodium $\geq 135 \mathrm{mmol} / \mathrm{L}$ at discharge were associated with mortality (OR $0.47,95 \% \mathrm{Cl} 0.23-0.94, P=0.034)$. Mortality rate fell with decreasing baseline s-sodium levels and was lower in the hyponatremia category $\leq 120 \mathrm{mmol} / \mathrm{L}$ vs $>120 \mathrm{mmol} / \mathrm{L}(14.8 \%$ and $27.8 \%, P<0.01)$. Patients with s-sodium level $\leq 120 \mathrm{mmol} / \mathrm{L}$ were more likely to have druginduced hyponatremia, whereas hypervolemic hyponatremia was more common in patients with s-sodium $>120 \mathrm{mmol} / \mathrm{L}$. Conclusion: Hyponatremia is associated with a substantial 1-year mortality, recurrence and rehospitalization rate. The positive correlation of s-sodium and mortality emphasizes the importance of the underlying disease, which determines the outcome besides hyponatremia itself.
\end{abstract}

\section{Introduction}

Hyponatremia is the most common electrolyte abnormality in hospitalized patients (1). Prevalence for mild hyponatremia is up to $30-42 \%$, depending on the health care setting and the patient population studied (2). Profound hyponatremia, defined as a serum sodium

www.eje-online.org DOI: 10.1530/EJE-16-0500
() 2016 European Society of Endocrinology Printed in Great Britain (s-sodium) level $<125 \mathrm{mmol} / \mathrm{L}$, is less common with a prevalence of $2-3 \%(3,4)$.

Hyponatremia is associated with an increased in-hospital, short-, and long-term mortality both in hospitalized $(5,6,7)$ and ambulatory patients $(8,9)$.

Published by Bioscientifica Ltd. 
An increased morbidity and mortality rate was shown especially in hyponatremic patients with medical conditions such as heart failure (10), myocardial infarction (11), liver cirrhosis (12), cancer (13), pneumonia (14) and stroke $(15,16)$.

Given the poor prognosis linked to hyponatremia, identification of modifiable factors in this context is of great interest. As highlighted by previous studies, hyponatremia per se may contribute to adverse outcome, which is reflected by a positive correlation of mortality and severity of hyponatremia $(17,18,19,20)$. Conversely, other studies observed a paradoxical fall in mortality rate as s-sodium values decreased below a certain level (e.g. $<120 \mathrm{mmol} / \mathrm{L})(5,19,21)$. This indicates the presence of factors other than hyponatremia itself affecting mortality, e.g. etiology of hyponatremia, comorbidities or therapeutic issues. So far, in previous publications, these possible outcome parameters have not been precisely assessed and studied in a prospective manner, and especially in profound hyponatremia, data are scarce and controversial $(7,19,21,22)$.

The aim of this study was to prospectively explore the predictive value of different clinical, laboratory and therapeutic parameters regarding outcome in a broad, well-defined cohort of hospitalized patients with profound hyponatremia.

\section{Methods}

\section{Study design}

From June 2011 to August 2013, 298 patients with profound hyponatremia presenting to the medical emergency department of the University Hospital Basel and the University Medical Clinic Aarau in Switzerland were included in a prospective observational study, which as a primary endpoint aimed to evaluate plasma copeptin as a diagnostic marker in the differential diagnosis of profound hyponatremia. As a predefined secondary endpoint, we aimed to explore clinical outcome and outcome predictors of patients with profound hyponatremia after 12 months of study entry. Inclusion criteria were full legal age, initial s-sodium level $\leq 125 \mathrm{mmol} / \mathrm{L}$ and a serum osmolality below $280 \mathrm{mmol} / \mathrm{kg}$. Patients with hyponatremia due to hyperglycemia or with age $<18$ years were excluded from the analysis. Study protocol was approved by the Ethics Committee of Basel and Aarau, and informed consent was obtained from all patients or in case of impaired mental state from their relatives.

\section{Baseline data collection}

Collected baseline data contained medical history, clinical examination (especially volume status) and specific laboratory testing including plasma and urine electrolytes, creatinine, osmolality, urea and uric acid. Furthermore, blood glucose, as well as thyroid-stimulating hormone and cortisol levels in unclear cases, were routinely measured.

\section{Diagnostic and therapeutic approach}

Initial diagnostic adjudication and treatment decisions were completely up to the attending physician and not influenced by the study team. Symptomatic hyponatremia was treated with hypertonic sodium chloride $(3 \% \mathrm{NaCl})$ infusion irrespective of the underlining cause. Oligo- or asymptomatic patients were treated with either fluid restriction or fluid administration $(0.9 \% \mathrm{NaCl}$ infusion) according to the cause of hyponatremia. Vaptans were not used, as they are not approved in Switzerland.

The final classification of hyponatremia was done by experienced board-certified endocrinologists (not involved in patients' treatment and care) in a standardized fashion using the above-mentioned data collected at admission. Moreover, information regarding management during hospitalization and treatment response was also included in the final diagnosis.

\section{Follow-up}

Follow-up was done by a structured telephone interview 12 months after study entry. Interviews were performed by study doctors, study nurses or students. Patients and/ or their respective family doctors were asked regarding rehospitalization and recurrent hyponatremia. Clinical report including laboratory findings was requested from every single rehospitalization to objectify information from phone interview and to get detailed information about circumstances of rehospitalization or eventually death.

\section{Outcome measures and analyzed outcome predictors}

We analyzed the influence of different laboratory and clinical parameters on three main outcomes: overall mortality, rehospitalization and recurrent profound hyponatremia. Besides 's-sodium level at admission', the following therapy-associated predictors were evaluated: 'ICU admission', 's-sodium correction rate' defined as 
delta s-sodium from admission to $24 \mathrm{~h}$ later, 'correction of hyponatremia' defined as s-sodium level $\geq 135 \mathrm{mmol} / \mathrm{L}$ at discharge, 'hospital aggravated hyponatremia' defined as decrease in s-sodium level $\geq 2 \mathrm{mmol} / \mathrm{L}$ during the first $48 \mathrm{~h}$ after hospital admission, and 'overly rapid correction' as a s-sodium increment $>12 \mathrm{mmol} / \mathrm{L}$ during first $24 \mathrm{~h}$ respectively $>18 \mathrm{mmol} / \mathrm{L}$ during first $48 \mathrm{~h}$. Furthermore, we analyzed whether etiology of hyponatremia or comorbidities assessed by the Charlson Comorbidity Index (CCI) were outcome predictors. The $\mathrm{CCI}$ is a comorbidity scoring system counting from 0 to 37 points, which allows to weigh disease severity according to the ICD-9-CM system $(23,24)$.

Because of controversial data regarding mortality according to hyponatremia severity, patients were divided in two categories (initial s-sodium level $\leq$ and $>120 \mathrm{mmol} / \mathrm{L}$ ) and were characterized in view of hyponatremia cause, therapy and outcome.

\section{Statistics}

For statistical analysis, all relevant clinical and laboratory parameters obtained by interview, clinical testing and reviewing of medical records were entered

Table 1 Baseline characteristics of 281 patients. Data are presented as \% ( $n$ ) and median (IQR: 25th-75th).

\begin{tabular}{l}
\hline Characteristics \\
\hline Age (years) \\
Female \\
Systolic blood pressure $(\mathrm{mmHg})$ \\
Heart rate (per minute) \\
Body temperature $\left({ }^{\circ} \mathrm{C}\right)$ \\
GCS (Glasgow Coma Score) \\
BMI (kg/m ${ }^{2}$ ) \\
Charlson Comorbidity Index \\
Etiology of hyponatremia \\
Malignant SIAD \\
Non-malignant SIAD \\
SIAD drugs/diuretics \\
SIAD idiopathic \\
SIAD lung \\
SIAD cns \\
Hypervolemic hyponatremia \\
Heart failure \\
Liver failure \\
Kidney failure \\
Hypovolemic hyponatremia \\
Primary polydipsia \\
Laboratory findings \\
S-sodium at hospital admission (mmol/L) \\
Plasma glucose (mmol/L) \\
\hline
\end{tabular}

\begin{tabular}{c}
\hline Values \\
\hline $72(61-80)$ \\
$66.5 \%(187)$ \\
$136(119-155)$ \\
$80(68-90)$ \\
$37(36.5-37.5)$ \\
$15(15-15)$ \\
$23.8(20.8-27.4)$ \\
$2(1-5)$ \\
$7.1 \%(20)$ \\
$54.1 \%(152)$ \\
$40.2 \%(113)$ \\
$4.6 \%(13)$ \\
$3.2 \%(9)$ \\
$6 \%(17)$ \\
$11.4 \%(32)$ \\
$5.3 \%(15)$ \\
$4.3 \%(12)$ \\
$1.8 \%(5)$ \\
$19.9 \%(56)$ \\
$7.5 \%(21)$ \\
$120(116-123)$ \\
$6.6(5.7-8.1)$ \\
\hline
\end{tabular}

BMI, body mass index. into an Excel database. Data were analyzed using STATA (12.1, Texas 77845, USA) and GraphPad Prism 6.00 for Windows (San Diego, California, USA). Discrete variables are expressed as frequency (percentage), continuous variables as mean with standard deviation (s.D.) and non-continuous variables as medians with interquartile range (IQR). For data not normally distributed, Mann-Whitney $U$ test was used for two-group comparison. Univariate and multivariate logistic regression analyses with two different models were performed. Both models were adjusted for age, CCI and s-sodium level at hospital admission. Model 1 was calculated for s-sodium $\geq 135 \mathrm{mmol} / \mathrm{L}$ at

Table 2 Follow-up and outcome data. Data are presented as $\%(n)$ and median (IQR: 25th-75th).

\begin{tabular}{|c|c|}
\hline \multicolumn{2}{|c|}{ Therapeutic findings during initial hospitalization } \\
\hline Delta s-sodium $24 \mathrm{~h}(\mathrm{mmol} / \mathrm{L})$ & $5(2-8)$ \\
\hline Overly rapid correction & $7.1 \%(20)$ \\
\hline Hospital aggravated hyponatremia & $8.5 \%(24)$ \\
\hline ICU admission & $36.7 \%(103)$ \\
\hline \multicolumn{2}{|l|}{ Laboratory findings at discharge } \\
\hline S-sodium (mmol/L) & $134(130-137)$ \\
\hline S-sodium $\geq 135 \mathrm{mmol} / \mathrm{L}$ & $49.1 \%(138)$ \\
\hline \multicolumn{2}{|l|}{ Outcome } \\
\hline Overall mortality & $20.6 \%(58)$ \\
\hline In-hospital-mortality & $3.9 \%(11)$ \\
\hline 30-day-mortality rate & $31 \%(18)$ \\
\hline Time to rehospitalization (days) & $75(21-206)$ \\
\hline 30-day-rehospitalization rate & $18.5 \%(52)$ \\
\hline Rehospitalization rate & $56.2 \%(158)$ \\
\hline $\begin{array}{l}\text { - with hyponatremia during first } \\
\text { rehospitalization }\end{array}$ & $60.8 \%(96)$ \\
\hline Recurrent rehospitalization & $28.5 \%(80)$ \\
\hline Recurrent hyponatremia & $42.7 \%(120)$ \\
\hline $\begin{array}{l}\text { Recurrent profound hyponatremia } \\
(\mathrm{Na} \leq 125 \mathrm{mmol} / \mathrm{L})\end{array}$ & $16 \%(45)$ \\
\hline \multicolumn{2}{|l|}{ Cause of Mortality $(n=58)$} \\
\hline Malignant disease & $32.8 \%(19)$ \\
\hline Unknown & $24.1 \%(14)$ \\
\hline Respiratory insufficiency & $12.1 \%(7)$ \\
\hline Heart failure & $10.3 \%(6)$ \\
\hline Septic shock & $8.6 \%(5)$ \\
\hline Liver failure & $8.6 \%(5)$ \\
\hline Renal failure & $3.4 \%(2)$ \\
\hline \multicolumn{2}{|l|}{ Cause of first rehospitalization $(n=158)$} \\
\hline Infectious & $18.4 \%(29)$ \\
\hline Orthopedic/surgery & $17.7 \%(28)$ \\
\hline Hematological/oncological & $11.4 \%(18)$ \\
\hline Others & $10.8 \%(17)$ \\
\hline Neurologic & $10.1 \%(16)$ \\
\hline Gastrointestinal & $9.5 \%(15)$ \\
\hline Cardiovascular & $7.6 \%(12)$ \\
\hline Hyponatremia & $6.3 \%(10)$ \\
\hline Psychiatric & $5.7 \%(9)$ \\
\hline Pulmonary & $2.5 \%(4)$ \\
\hline
\end{tabular}

BMI, body mass index. 
Table 3 Univariate analysis.

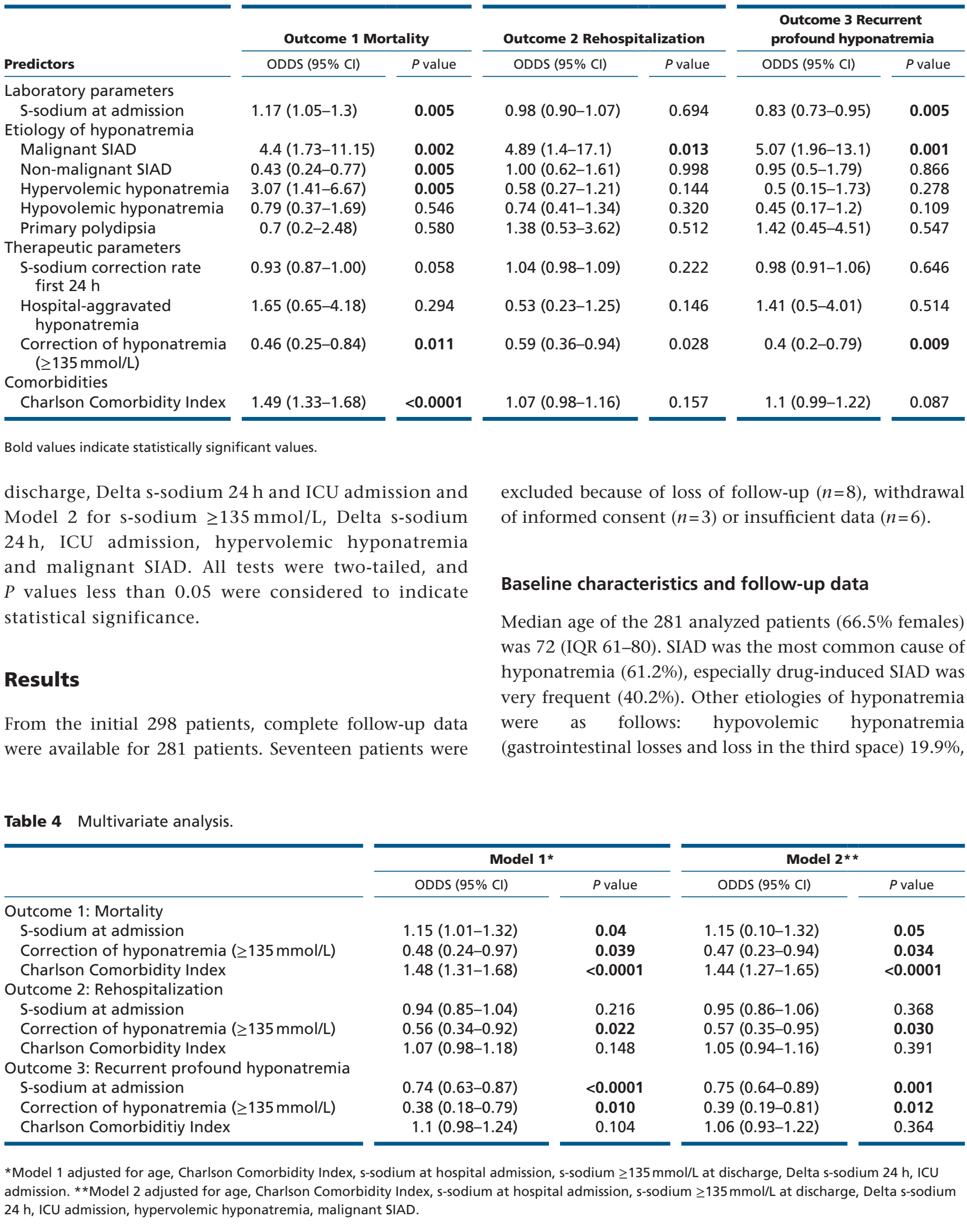


hypervolemic hyponatremia (liver, heart and kidney failure) $11.4 \%$ and primary polydipsia $7.5 \%$.

Median s-sodium level at hospital admission was $120 \mathrm{mmol} / \mathrm{L}$ (IQR 116-123) and was raised by a median of $5 \mathrm{mmol} / \mathrm{L}$ (IQR 2-8) during the first $24 \mathrm{~h}$. Half of the patients (49.1\%) reached normal s-sodium levels $(\geq 135 \mathrm{mmol} / \mathrm{L})$ at discharge. During hospitalization, 36.7\% of patients were admitted to the intensive care unit (ICU) and $8.5 \%$ were treated with $3 \% \mathrm{NaCl}$ infusion. Hospital aggravated hyponatremia occurred in $8.5 \%$ of patients. Overly rapid correction was seen in 20 (7.1\%) patients, whereby nine of them received $0.9 \% \mathrm{NaCl}$ infusion, six $3 \% \mathrm{NaCl}$ infusion and five patients were treated with fluid restriction. No patient developed osmotic demyelination syndrome (ODS) during the observation phase. Detailed baseline characteristics are shown in Table 1 and follow-up data in Table 2.

Table 5 Characteristics of patients with initial s-sodium level of $\leq 120 \mathrm{mmol} / \mathrm{L}$ and $>120 \mathrm{mmol} / \mathrm{L}$. Data are presented as $\%(n)$ and median (IQR: 25th-75th).

\begin{tabular}{l}
\hline Characteristics \\
\hline Age (years) \\
Female \\
Systolic blood pressure (mmHg) \\
Heart rate (per minute) \\
Charlson Comorbidity Index \\
Etiology of hyponatremia \\
Malignant SIAD \\
Non-malignant SIAD \\
SIAD drugs/diuretics \\
SIAD idiopathic \\
SIAD lung \\
SIAD cns \\
Hypervolemic hyponatremia \\
Heart failure \\
Liver failure \\
Kidney failure \\
Hypovolemic hyponatremia \\
Primary polydipsia \\
Medication \\
Diuretics \\
Thiazide diuretics \\
Loop-diuretics \\
Potassium-sparing diuretics \\
Antihypertensives \\
NSAID \\
Neuroleptics \\
Opioids \\
Laboratory findings \\
S-sodium at hospital admission (mmol/L) \\
S-sodium at discharge (mmol/L) \\
S-sodium $\geq 135$ mmol/L at discharge \\
Therapeutic findings \\
Delta s-sodium $24 \mathrm{~h}$ (mmol/L) \\
Delta s-sodium $48 \mathrm{~h}$ (mmol/L) \\
Overly rapid correction \\
ICU admission \\
3\% NaCl infusion \\
Outcome \\
Rechospitl mortality \\
Recurrent profound hyponatremia \\
\hline
\end{tabular}

\begin{tabular}{c}
\hline S-sodium $\leq \mathbf{1 2 0} \mathbf{~ m m o l} / \mathbf{L}(n=155)$ \\
\hline $73(62-81)$ \\
$65.8 \%(102)$ \\
$139(120-159)$ \\
$76(65.8-88)$ \\
$2(1-4)$
\end{tabular}

$7.7 \%(12)$

$62.6 \%$ (97)

$49 \%(76)$

$5.2 \%(8)$

$3.2 \%(5)$

$5.2 \%(8)$

$7.7 \%(12)$

$3.9 \%(6)$

$1.9 \%$ (3)

$1.9 \%$ (3)

$15.5 \%(24)$

$6.5 \%(10)$

$64.5 \%(100)$

$50.3 \%(78)$

$16.1 \% \%$ (25)

$12.3 \%$ (19)

$54.2 \%$ (84)

$11 \%(17)$

$10.3 \%(16)$

$7.7 \%(12)$

$117(113-120)$
$135(131-137)$

$52.3 \%(81)$

$$
\begin{gathered}
5(2-9) \\
11(7-15) \\
11.6 \%(18) \\
48.4 \%(75) \\
12.3 \%(19)
\end{gathered}
$$

$14.8 \%(23)$

$1.3 \%(2)$

$56.1 \%(87)$

$45.8 \%(71)$

$21.3 \%(33)$

\begin{tabular}{c}
\hline S-sodium $>\mathbf{1 2 0} \mathbf{~ m m o l} / \mathbf{L}(n=126)$ \\
\hline $70(58-79.3)$ \\
$67.5 \%(85)$ \\
$135(114-153)$ \\
$84(70-97)$ \\
$2(1-5.3)$
\end{tabular}

\begin{tabular}{l}
$P$ value \\
\hline 0.0769 \\
0.7712 \\
0.0811 \\
0.0008 \\
0.0436
\end{tabular}

$6.3 \%(8)$

0.6533

$43.7 \%$ (55)

$29.4 \%$ (37)

$4 \%(5)$

0.0016

0.0008

0.6379

$3.2 \%$ (4)

$7.1 \%(9)$

0.9826

0.4903

$15.9 \%$ (20)

0.0333

$7.1 \%$ (9)

$7.1 \%$ (9)

0.2265

$1.6 \%$ (2)

$25.4 \%$ (32)

$8.7 \%(11)$

0.8290

0.0390

0.4718

$54 \%(68)$

0.0736

$37.3 \%(47)$

0.0293

$19.8 \%(25)$

0.4199

0.9292

0.1363

0.3068

$15.1 \%$

$7.9 \%(10)$

$11.9 \%(15)$

0.4941

0.2404

$\begin{array}{cr}123(122-124) & <0.0001 \\ 134(129-137) & 0.2306 \\ 45.2 \%(57) & 0.2429 \\ & \\ 4.5(1-6) & 0.0163 \\ 8(3.5-11) & <0.0001 \\ 1.6 \%(2) & 0.0012 \\ 22.2 \%(28) & <0.0001 \\ 4 \%(5) & 0.0136 \\ 27.8 \%(35) & \\ 7.1 \%(9) & 0.0078 \\ 56.3 \%(71) & 0.0121 \\ 38.9 \%(49) & 0.9712 \\ 9.5 \%(12) & 0.2449 \\ & 0.0076\end{array}$

BMI, body mass index. 


\section{Outcome measures}

\section{Mortality}

Overall mortality was $20.6 \%$, whereof $3.9 \%$ died during the initial hospital stay and $31 \%$ within the first 30 days after discharge. Leading causes of death were malignant disease $(32.8 \%)$, respiratory $(12.1 \%)$ respectively cardiac arrest $(10.3 \%)$, or unspecified polymorbidity (24.1\%) (Table 2). In none of these patients, death could directly be attributed to hyponatremia, as suggestive hyponatremia complications (e.g. brain edema, epileptic seizure) were not recorded.

Mortality risk was significantly higher in patients with multiple comorbidities expressed by a high CCI both in univariate and multivariate analysis (OR 1.49, 95\% CI 1.33-1.68, $P<0.001$ and OR 1.45, 95\% CI $1.27-1.65, P<0.001)$.

A significant correlation with mortality was shown for 's-sodium level at admission', whereby higher values were associated with a higher mortality rate (OR 1.17, 95\% CI 1.1-1.3, $P=0.005)$. This association was also present in multivariate analysis (OR 1.14, 95\% CI 1.01-1.29, $P=0.036$ ).

Single therapeutic factors, such as 'correction rate', 'ICU admission' or 'hospital aggravated hyponatremia', were not associated with mortality. However, correction of hyponatremia (s-sodium level $\geq 135 \mathrm{mmol} / \mathrm{L}$ ) was significantly associated with a lower mortality rate (OR 1.09, 95\% CI 1.01-1.18, $P=0.02$ and OR 0.46, 95\% CI $0.3-0.8, P=0.011$ ), even after multivariate adjustment (OR $0.47,95 \%$ CI $0.23-0.95, P=0.034)$.

Concerning etiology of hyponatremia, higher mortality rates were found for hypervolemic hyponatremia and malignant SIAD (OR 3.07, 95\% CI 1.4-6.7, $P=0.005$ and OR 4.4, 95\% CI 1.7-11.2, $P=0.002$ respectively). On the contrary, non-malignant SIAD was associated with a lower mortality rate (OR $0.45,95 \%$ CI $0.24-0.77$, $P=0.005)$. However, after multivariate analysis, etiologies of hyponatremia lost significance as outcome markers (data not shown). Detailed results for univariate and multivariate analyses are shown in Tables 3 and 4 .

\section{Rehospitalization and recurrent hyponatremia}

Thirty-day rehospitalization rate was $18.5 \%$, but $56.2 \%$ of patients were rehospitalization at least once in 12 months, $28.5 \%$ repeatedly. During follow-up, recurrent hyponatremia was observed in $42.7 \%$ of patients, being profound (s-sodium level $\leq 125 \mathrm{mmol} / \mathrm{L}$ ) again in $16 \%$ (Table 2).
'S-sodium level at admission' was not linked to an increased rehospitalization rate, but showed a significant association with recurrent profound hyponatremia, after multivariate adjustment (OR 0.75, 95\% CI 0.64-0.89, $P=0.001)$ also.

The risk of rehospitalization and recurrent profound hyponatremia was lower in patients who reached eunatremia at discharge ('correction of hyponatremia'), both in univariate and multivariate analysis (OR 0.57, 95\% CI $0.35-0.95, \quad P=0.03$ and OR $0.39, \quad 95 \%$ CI $0.19-0.81, P=0.012$ respectively).

'Malignant SIAD' was a risk factor for rehospitalization (OR 4.89, 95\% CI 1.4-17.1, $P=0.013)$ and recurrent profound hyponatremia (OR 5.07, 95\% CI 1.96-13.1, $P=0.001$ ) in univariate but not in multivariate analysis.

All other variables were not significantly associated with the outcomes of rehospitalization and recurrent profound hyponatremia (Tables 3 and 4).

\section{Patients characteristics according to hyponatremia categories: s-sodium $\leq$ and $>120 \mathrm{mmol} / \mathrm{L}$}

Characteristics of patients with initial s-sodium level $\leq$ vs $>120 \mathrm{mmol} / \mathrm{L}$ revealed several differences (Table 5).

In-hospital and overall mortality was higher in patients with initial s-sodium levels $>120 \mathrm{mmol} / \mathrm{L}(7.1 \%$ vs $1.3 \%, P=0.0121$ vs $27.8 \%$ vs $14.8 \%, P=0.0078$ ). Figure 1 shows Kaplan-Meier survival curves for both subgroups. Concerning etiology of hyponatremia patients with a s-sodium level $>120 \mathrm{mmol} / \mathrm{L}$ more

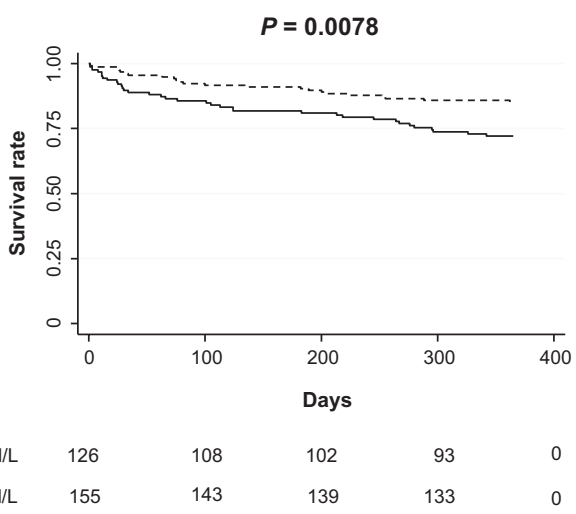

$\begin{array}{lrrrrr}\text { s-sodium >120mmol/L } & 126 & 108 & 102 & 93 & 0 \\ \text { s-sodium } \leq 120 \mathrm{mmol} / \mathrm{L} & 155 & 143 & 139 & 133 & 0\end{array}$

s-sodium $>120 \mathrm{mmol} / \mathrm{L} \quad$ - - - - s s-sodium $\leq 120 \mathrm{mmol} / \mathrm{L}$

Figure 1

Kaplan-Meier curve. Survival rate in patients with initial s-sodium level $\leq 120 \mathrm{mmol} / \mathrm{L}$ and $>120 \mathrm{mmol} / \mathrm{L}$. 
often suffered from hypervolemic or hypovolemic hyponatremia $(15.9 \%$ vs $7.7 \%, P=0.0333$ and $25.4 \%$ vs $15.5 \%, P=0.039$ respectively). With $62.6 \%$, nonmalignant SIAD - especially due to drugs/diuretics was the most frequent etiology in the lower s-sodium subgroup (49\% vs $29.4 \%, P=0.0008$ ).

Initial s-sodium correction rate was higher when hyponatremia was more pronounced: $5 \mathrm{mmol} / \mathrm{L}$ during the first $24 \mathrm{~h}$ compared with $4.5 \mathrm{mmol} / \mathrm{L}(P=0.0163)$ in the subgroup with initial s-sodium level $>120 \mathrm{mmol} / \mathrm{L}$. Furthermore, ICU admission and treatment with 3\% $\mathrm{NaCl}$ infusion was more common in patients with initial s-sodium $\leq 120 \mathrm{mmol} / \mathrm{L}$ (48.4\% vs $22.2 \%, P<0.0001$ and $12.3 \%$ vs $4 \%, P=0.0136$ respectively).

During follow-up, patients in the lower hyponatremia category recurred more often with profound hyponatremia $(21.3 \%$ vs $9.5 \%, P=0.0076)$. On the contrary, rehospitalization rate was comparable between the two groups $(56.1 \%$ vs $56.3 \%, P=0.97)$.

\section{Discussion}

We herein confirm the adverse outcomes linked to hyponatremia. As a key finding, we observed a highly significant association of the CCI and death, meaning that mortality in hyponatremia is mainly influenced by comorbidities. Besides CCI, we identified two other important predictors of long-term survival: 's-sodium level at admission' and 'correction of hyponatremia'.

The mortality rate in our cohort of hyponatremic patients was $20.6 \%$, which is well within the published range of $5-50 \%(5,19,2)$. Supposing that hyponatremia itself is causing death, an inverse relationship between s-sodium and mortality would be expected. In contrast, we found a strong, positive association between initial s-sodium concentration and mortality; thus, mortality rate was lower in patients with lower s-sodium levels. This is in contrast with a recently published meta-analysis indicating that hyponatremia-related risk augments with decreasing s-sodium level (6). In this meta-analysis, only 5 of 81 analyzed studies used a s-sodium cutoff of $<125 \mathrm{mmol} / \mathrm{L}$ as was used in our study, thus supporting an inverse correlation of hyponatremia level and death in mild but not in profound hyponatremia. Indeed, similar to our data, other studies observed a paradoxical fall in mortality rate in profound hyponatremia $(5,19,21,25)$. In two large prospective studies, one-year mortality rate was highest in patients with a s-sodium between 120 and $129 \mathrm{mmol} / \mathrm{L}$ and lower in patients with a s-sodium
$>130 \mathrm{mmol} / \mathrm{L}$ or $<120 \mathrm{mmol} / \mathrm{L}$ respectively $(5,19)$. As a possible explanation for the lower mortality rate in patients with lower s-sodium levels, we found a lower CCI and in line with a previous study, significantly more cases with non-malignant (mainly drug induced) SIAD in the lower hyponatremia category $(19,21)$. Thus, etiologies of hyponatremia may vary according to its degree and notably drug-induced SIAD seems to predispose to very low s-sodium levels (26). We assume that hyponatremia being a marker of disease severity and outcome in heart failure or liver cirrhosis - might not be similarly linked to mortality in other conditions like drug-induced SIAD.

An alternative rational for the lower mortality in patients with s-sodium $<120 \mathrm{mmol} / \mathrm{L}$ may be a different therapeutic approach in very profound vs milder hyponatremia. Indeed, we observed a higher percentage of ICU admission and of hypertonic saline administration as well as a more rapid initial sodium correction rate in patients with s-sodium of $\leq 120$ vs $>120 \mathrm{mmol} / \mathrm{L}$. Furthermore, in patients with s-sodium levels $>120 \mathrm{mmol} / \mathrm{L}$, hospital-aggravated hyponatremia tended to be more frequent suggesting a delayed or inadequate management of hyponatremia. This is in accordance with the recently published Hyponatremia Registry showing that active treatment significantly depends on hyponatremia severity (27). However, the hypothesis that modality and velocity of hyponatremia treatment influences outcome could not be strengthened by our data: besides hyponatremia correction, we did not find a significant association of single therapeutic factors and mortality - probably because of insufficient power.

Hyponatremia correction was reached in 49.1\% of our patients and strongly correlated with a lower mortality rate. This is in agreement with a recent meta-analysis, finding a reduced risk of death for any degree of hyponatremia improvement (28). One might postulate that patients who reach normonatremia are generally healthier, and their condition is more often attributable to a transient etiology of hyponatremia (e.g. drug-induced SIAD, pulmonary infection) (26). However, when looking at patients in the hyponatremia category $\leq 120 \mathrm{mmol} / \mathrm{L}$ - harboring a lower CCI and often a transient hyponatremia cause - we did not find a higher percentage of hyponatremia correction. This is probably explained by an 'earlier' hospital discharge of healthier patients (before reaching an s-sodium level of $\geq 135 \mathrm{mmol} / \mathrm{L}$ ).

In the aforementioned meta-analysis, comorbidities were not considered as confounders due to insufficient data. In our study, data were adjusted for age, comorbidities 
(CCI) and cause of hyponatremia, but 'correction of hyponatremia' remained significantly associated with a reduced mortality rate. This underscores the significance of hyponatremia correction across different clinical conditions, which could play an important role in preventing mortality.

The 1-year readmission rate in our study was very high (56.2\%). Not surprisingly, malignant SIAD turned out to be a predictor of both rehospitalization and rehyponatremia. Reasons for rehospitalization were various, but hyponatremia itself was only rarely indicated as the main cause. However, many patients presented with accompanying hyponatremia, and it is tempting to speculate that it was a contributing factor for rehospitalization. This is supported by the fact that CCI was not a predictor of readmission, whereas correction of hyponatremia was significantly associated with a lower rehospitalization rate even after multivariate adjustment.

Interestingly, there was a significant association of hyponatremia severity and recurrent profound hyponatremia; patients with initial s-sodium level $\leq 120 \mathrm{mmol} / \mathrm{L}$ recurred more often compared with those with initial levels above $120 \mathrm{mmol} / \mathrm{L}$. In these patients with mainly drug-induced SIAD, this may be explained by a restart of predisposing drugs after normalization of hyponatremia.

Our study has the following strengths. Aside from large-scale epidemiologic studies, it is the largest reported series of prospectively enrolled patients with profound hyponatremia. The prospective study design with specific testing allowed us to carefully categorize patients into diagnostic groups. Excluding patient with hyperglycemiainduced hyponatremia eliminated an important and frequent confounder in hyponatremia studies. However, some limitations should be considered. First, this is a predefined secondary analysis of a prospective study. Thus, there was no power calculation specific to this paper, what may weaken our findings. Secondly, when focusing on the subgroups on initial s-sodium level $\leq$ or $>120 \mathrm{mmol} / \mathrm{L}$, the primary endpoint death was reached in 'only' 23 and 35 patients respectively. Thus, caution is needed when interpreting results according to these hyponatremia categories. Furthermore, although in line with previous studies, the division into these two categories at a cutoff of $120 \mathrm{mmol} / \mathrm{L}$ is somehow arbitrary.

In conclusion, we confirm the impact of profound hyponatremia in daily clinical practice, with a high 1-year mortality, recurrence and rehospitalization rate. The positive correlation of the CCI and initial s-sodium level with mortality emphasizes the importance of the underlying disease, which seems to particularly impact on prognosis. But still, hyponatremia of any degree is associated with poor outcome, and our data suggest that a particular attention should be paid to hyponatremia itself as a therapeutic target - irrespective of etiology and severity.

Declaration of interest

The authors declare that there is no conflict of interest that could be perceived as prejudicing the impartiality of the research reported.

\section{Funding}

The study was investigator initiated and primarily supported by the University Hospital Basel, Basel University, Medical University Clinic Aarau and the Swiss National Foundation research funds. M C-C was supported by a grant from the Swiss National Foundation (PP00P3-123346), Switzerland.

\section{Acknowledgments}

The authors thank the staff of the clinics and departments of emergency medicine, internal medicine, and the department of endocrinology, diabetology and metabolism for most helpful support during study. Furthermore, the authors thank the many supporters, study and laboratory personnel at both participating centers, especially Cemile Bathelt, Kathrin Ulrich, Merih Guglielmetti, Sonja Schwenne, Kristina Schumacher, Renate Hunziker and Ursina Minder. They also thank the members of the OPTIMA study team for their support for patient enrolment and data collection.

\section{References}

1 Spasovski G, Vanholder R, Allolio B, Annane D, Ball S, Bichet D, Decaux G, Fenske W, Hoorn EJ, Ichai C et al. Clinical practice guideline on diagnosis and treatment of hyponatraemia. Nephrology Dialysis Transplantation 201429 (Supplement 2) i1-i39. (doi:10.1093/ ndt/gfu040)

2 Upadhyay A, Jaber BL \& Madias NE. Incidence and prevalence of hyponatremia. American Journal of Medicine 2006119 S30-S35. (doi:10.1016/j.amjmed.2006.05.005)

3 Upadhyay A, Jaber BL \& Madias NE. Epidemiology of hyponatremia. Seminars in Nephrology 200929 227-238. (doi: 10.1016/j.semnephrol. 2009.03.004)

4 Hawkins RC. Age and gender as risk factors for hyponatremia and hypernatremia. Clinica Chimia Acta 2003337 169-172. (doi:10.1016/j.cccn.2003.08.001)

5 Waikar SS, Mount DB \& Curhan GC. Mortality after hospitalization with mild, moderate, and severe hyponatremia. American Journal of Medicine 2009122 857-865. (doi:10.1016/j.amjmed.2009.01.027)

6 Corona G, Giuliani C, Parenti G, Norello D, Verbalis JG, Forti G, Maggi M \& Peri A. Moderate hyponatremia is associated with increased risk of mortality: evidence from a meta-analysis. PLOS ONE 20138 e80451. (doi:10.1371/journal.pone.0080451)

7 Balling L, Gustafsson F, Goetze JP, Dalsgaard M, Nielsen H, Boesgaard S, Bay M, Kirk V, Nielsen OW, Kober L et al. Hyponatraemia at hospital admission is a predictor of overall mortality. Internal Medicine Journal 201545 195-202. (doi:10.1111/imj.12623)

8 Gankam-Kengne F, Ayers C, Khera A, de Lemos J \& Maalouf NM. Mild hyponatremia is associated with an increased risk of death in an ambulatory setting. Kidney International 201383 700-706. (doi:10.1038/ki.2012.459)

9 Sajadieh A, Binici Z, Mouridsen MR, Nielsen OW, Hansen JF \& Haugaard SB. Mild hyponatremia carries a poor prognosis in 
community subjects. American Journal of Medicine 2009122 679-686. (doi:10.1016/j.amjmed.2008.11.033)

10 Gheorghiade M, Abraham WT, Albert NM, Gattis Stough W, Greenberg BH, O'Connor CM, She L, Yancy CW, Young J \& Fonarow GC. Relationship between admission serum sodium concentration and clinical outcomes in patients hospitalized for heart failure: an analysis from the OPTIMIZE-HF registry. European Heart Journal 200728 980-988. (doi:10.1093/eurheartj/ehl542)

11 Singla I, Zahid M, Good CB, Macioce A \& Sonel AF. Effect of hyponatremia $(<135 \mathrm{mEq} / \mathrm{L})$ on outcome in patients with non-STelevation acute coronary syndrome. American Journal of Cardiology 2007100 406-408. (doi: 10.1016/j.amjcard.2007.03.039)

$12 \mathrm{Yu}$ C, Sharma N \& Saab S. Hyponatremia: clinical associations, prognosis, and treatment in cirrhosis. Experimental and Clinical Transplantation 201311 3-11. (doi:10.6002/ect.2012.0147)

13 Doshi SM, Shah P, Lei X, Lahoti A \& Salahudeen AK. Hyponatremia in hospitalized cancer patients and its impact on clinical outcomes. American Journal of Kidney Diseases 201259 222-228. (doi:10.1053/ j.ajkd.2011.08.029)

14 Nair V, Niederman MS, Masani N \& Fishbane S. Hyponatremia in community-acquired pneumonia. American Journal of Nephrology 2007 27 184-190. (doi:10.1159/000100866)

15 Huang WY, Weng WC, Peng TI, Chien YY, Wu CL, Lee M, Hung CC \& Chen KH. Association of hyponatremia in acute stroke stage with three-year mortality in patients with firstever ischemic stroke. Cerebrovascular Diseases 201234 55-62. (doi:10.1159/000338906)

16 Rodrigues B, Staff I, Fortunato G \& McCullough LD. Hyponatremia in the prognosis of acute ischemic stroke. Journal of Stroke and Cerebrovascular Diseases 201423 850-854. (doi:10.1016/ j.jstrokecerebrovasdis.2013.07.011)

17 Wald R, Jaber BL, Price LL, Upadhyay A \& Madias NE. Impact of hospital-associated hyponatremia on selected outcomes. Archives of Internal Medicine 2010170 294-302. (doi:10.1001/ archinternmed.2009.513)

18 Bennani SL, Abouqal R, Zeggwagh AA, Madani N, Abidi K, Zekraoui A \& Kerkeb O. Incidence, causes and prognostic factors of hyponatremia in intensive care. Revue de Médecine Interne 200324 224-229.
19 Holland-Bill L, Christiansen CF, Heide-Jorgensen U, Ulrichsen SP, Ring T, Jorgensen JO \& Sorensen HT. Hyponatremia and mortality risk: a Danish cohort study of 279508 acutely hospitalized patients. European Journal of Endocrinology 2015173 71-81. (doi:10.1530/EJE15-0111)

20 Asadollahi K, Hastings IM, Beeching NJ \& Gill GV. Laboratory risk factors for hospital mortality in acutely admitted patients. QJM 2007 100 501-507. (doi:10.1093/qjmed/hcm055)

21 Chawla A, Sterns RH, Nigwekar SU \& Cappuccio JD. Mortality and serum sodium: do patients die from or with hyponatremia? Clinical Journal of the American Society of Nephrology 20116 960-965. (doi:10.2215/CJN.10101110)

22 Baran D \& Hutchinson TA. The outcome of hyponatremia in a general hospital population. Clinical Nephrology 198422 72-76.

23 Charlson ME, Pompei P, Ales KL \& MacKenzie CR. A new method of classifying prognostic comorbidity in longitudinal studies: development and validation. Journal of Chronic Diseases $1987 \mathbf{4 0}$ 373-383. (doi:10.1016/0021-9681(87)90171-8)

24 Deyo RA, Cherkin DC \& Ciol MA. Adapting a clinical comorbidity index for use with ICD-9-CM administrative databases. Journal of Clinical Epidemiology 199245 613-619. (doi:10.1016/08954356(92)90133-8)

25 Clayton JA, Le Jeune IR \& Hall IP. Severe hyponatraemia in medical in-patients: aetiology, assessment and outcome. QJM 200699 505-511. (doi:10.1093/qjmed/hcl071)

26 Shepshelovich D, Leibovitch C, Klein A, Zoldan S, Milo G, Shochat T, Rozen-Zvi B, Gafter-Gvili A \& Lahav M. The syndrome of inappropriate antidiuretic hormone secretion: Distribution and characterization according to etiologies. European Journal of Internal Medicine 201526 819-824. (doi:10.1016/j.ejim.2015.10.020)

27 Greenberg A, Verbalis JG, Amin AN, Burst VR, Chiodo JA 3rd, Chiong JR, Dasta JF, Friend KE, Hauptman PJ, Peri A et al. Current treatment practice and outcomes. Report of the hyponatremia registry. Kidney International 201588 167-177. (doi: 10.1038/ ki.2015.4)

28 Corona G, Giuliani C, Verbalis JG, Forti G, Maggi M \& Peri A. Hyponatremia improvement is associated with a reduced risk of mortality: evidence from a meta-analysis. PLOS ONE 201510 e0124105. (doi:10.1371/journal.pone.0124105)

Received 11 June 2016

Revised version received 19 August 2016

Accepted 1 September 2016 